Received: 2014.02.18 Accepted: 2014.03.14 Published: 2014.06.28

Authors' Contribution: Study Design A Data Collection B Statistical Analysis C Data Interpretation D Manuscript Preparation E Literature Search F Funds Collection $\mathrm{G}$

\section{Obturator hernia: A diagnostic challenge}

1 Department of General Medicine, Saga University Hospital, Saga City, Japan 2 The Community Center of General Medicine, Saga University Hospital, Saga City, Japan
Corresponding Author: Conflict of interest:

\begin{abstract}
Patient: Female, 90
Final Diagnosis:

Symptoms:

Medication:

Clinical Procedure:

Specialty:

Objective:

Background:

Case Report:

Conclusions:

Obturator hernia

Epigastric pain • vomiting

$-$

\section{Gastroenterology and Hepatology}

\section{Challenging differential diagnosis}

Obturator hernia $(\mathrm{OH})$ can be difficult to diagnose because it shows only nonspecific signs and symptoms. Although pain in a lower limb caused by compression of the obturator nerve by the hernia in the obturator canal (Howship-Romberg sign) is a characteristic sign, its presence is rather rare.

We herein describe the case of a 90-year-old woman with an $\mathrm{OH}$ that was difficult to diagnose because of her slight abdominal signs and symptoms on admission and subtle abdominal computed tomography (CT) findings. Although the $\mathrm{CT}$ images revealed the presence of an $\mathrm{OH}$, this finding was overlooked because it contained only a part of the small intestine wall, which is called the Richter type. Fortunately, her condition improved dramatically with only conservative treatment.

Although early diagnosis is essential to reduce morbidity and mortality, $\mathrm{OH}$ can be a diagnostic challenge even with abdominal CT.
\end{abstract}

MeSH Keywords:

Full-text PDF:

Geriatrics • Hernia - diagnosis • Ileus - diagnosis

http://www.amjcaserep.com/download/index/idArt/890555 


\section{Background}

Obturator hernia $(\mathrm{OH})$ can be difficult to diagnose because it is an extremely rare type of abdominal hernia and is only associated with nonspecific signs and symptoms. Typical patients with an $\mathrm{OH}$ are thin, elderly women. Although the HowshipRomberg sign is rather specific among various symptoms, this sign is rare and still non-pathognomonic. Computed tomography (CT) is helpful in the diagnosis of $\mathrm{OH}[1,2]$. $\mathrm{CT}$ is not a perfect tool, however, and several reports have shown the sensitivity of CT to be around $90 \%[1,3]$.

We herein present a case of $\mathrm{OH}$ that was difficult to diagnose because of the patient's subtle signs and symptoms and very elusive $\mathrm{CT}$ findings.

\section{Case Report}

A 90-year-old woman with a history of hypertension and severe dementia experienced epigastric pain and vomiting after dinner. Her family sought care from a primary care physician. She was suspected to have a myocardial infarction because of her ambiguous complaints of epigastric pain and abnormal portable electrocardiogram findings. She was immediately transferred to the emergency department of our hospital by ambulance. She was initially seen by a cardiologist, who ruled-out the presence of ischemic heart disease. She was then referred to the general medicine department. She only complained of nausea and epigastric discomfort without abdominal pain. On physical examination, her vital signs were stable. Her abdomen was soft and flat, and her bowel sounds were present but weak. On palpation, she had tenderness on pressure in the lower abdomen without peritoneal irritability. Peripheral blood analysis revealed a white blood cell count of $10,200 / \mu l$ without a left shift. Her C-reactive protein level was $0.10 \mathrm{mg} / \mathrm{dl}$. Her abdominal X-ray showed a dilatation of the small intestine in the left lower abdomen without air-fluid levels. Dilatation of the colon was not detected. Although she had no history of previous abdominal surgery, we could not rule-out the possibility of mild mechanical ileus, despite these minor findings.

While in the hospital, she was not allowed to eat, and intravenous fluids were administered. However, she continued to have abdominal tenderness in the lower abdomen. Furthermore, she expelled no gas or stool after her admission. On the third hospital day, a follow-up abdominal X-ray showed increased small intestinal dilatation and air-fluid levels (Figure 1A). For the first time after admission, she was examined by abdominal CT. Although it also showed intestinal dilatation similar to her abdominal X-ray, the enhancement of the intestinal wall was good, which meant that circulation in the intestinal wall was not impaired. In addition, there was no elevation of CK or $\mathrm{LDH}$ in her blood test result. We installed an ileus tube, and her symptoms improved dramatically. Radiological enteroclysis showed several apparently constricted areas, which we considered to be the cause of her ileus. We explored the cause of the constriction, considering intestinal tuberculosis or Crohn's disease. However, the level of QuantiFERON TB-2G was normal. There was no abnormal finding in her gastrointestinal endoscopy or total colon endoscopy. When we reviewed the abdominal CT scan, we found a slight obturator hernia in her right obturator canal, which contained only a part of the small intestine wall (Figure 1B), called the Richter type [4]. Although $\mathrm{OH}$ requires surgery, we selected the conservative treatment because her condition had already been improving without surgery. Furthermore, we thought that she could not be placed at rest after the operation and had many risks for surgery due to her old age and dementia, because of which her family wanted to avoid preventive surgery. She was discharged on day 52 of hospitalization without having undergone surgery. No recurrence of the hernia was noted during the 6-month follow-up.

\section{Discussion}

$\mathrm{OH}$ is a rare condition that can cause ileus and usually requires emergency surgery [1-5]. The associated symptoms and signs are nonspecific, such as nausea and vomiting (63\%), abdominal or groin pain (57\%), or a palpable lump (10\%) [1]. Among them, the Howship-Romberg sign is rather specific. It is defined as a pain in the lower limbs caused by compression of the obturator nerve by the hernia in the obturator canal. Some studies have reported that the Howship-Romberg sign is seen in $37 \%$ to $60 \%$ of cases [6-8]. However, $\mathrm{OH}$ tends to develop in thin, aged women, who cannot describe their symptoms due to dementia. In fact, our patient, who had dementia, had difficulty expressing her symptoms, which made it extremely challenging to diagnose $\mathrm{OH}$ correctly.

We performed radiological enteroclysis to diagnose the cause of her bowel obstruction. However, this often shows only nonspecific, stricture-like findings and fails to reveal the presence of $\mathrm{OH}$ [9]. Furthermore, false-positive findings are reported. If the cause of her ileus was truly small bowel constriction revealed by radiological enteroclysis, her small bowel obstruction would not have improved with only conservative treatment. Although ultrasonography is widely used to show the presence of bowel obstruction, the $\mathrm{OH}$ itself was difficult to detect by ultrasonography [2]. Ultrasonography has no advantage over $\mathrm{CT}$, because its specificity in diagnosis of $\mathrm{OH}$ is only about 30\% [10]. It may be difficult to make the correct diagnosis using only ultrasonography, especially in a patient like ours, with a very small hernia. Abdominal CT is regarded as a standard diagnostic modality for $\mathrm{OH}$, with a high accuracy of 

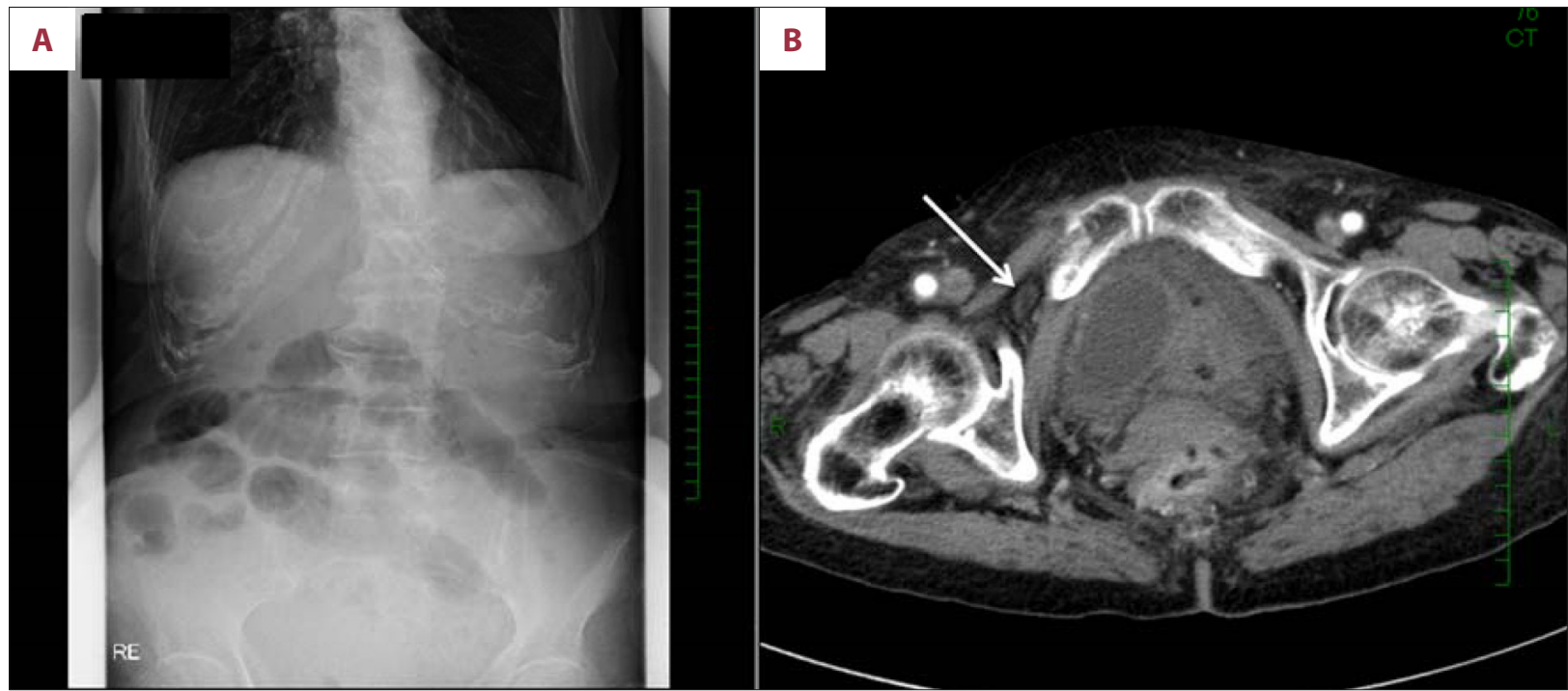

Figure 1. (A) Abdominal X-ray reveals marked dilatation of the small intestine and air-fluid levels. Gas is not detected in the colon. (B) Abdominal CT shows a small obturator hernia in the right thigh containing only a part of the wall of small intestine (arrow).

$>90 \%$ [1]. Abdominal CT can reveal incarceration of the intestine into the obturator canal, which is typically shown as a luminal structure with intestinal gas. However, even abdominal CT is not a perfect modality, and its use does not decrease the rate of bowel necrosis or postoperative morbidity and mortality [7]. We overlooked the presence of $\mathrm{OH}$ in our patient because of very subtle findings of $\mathrm{OH}$ on $\mathrm{CT}$, showing only a part of the small intestine wall in the hernia (Richter type).

Nasir et al. reported that $53 \%$ of patients with $\mathrm{OH}$ had an incarcerated hernia. Of these, $47 \%$ contained strangulated bowels [1]. Furthermore, Rodriguez-Hermosa et al. reported that the rate of strangulated hernias was $75 \%$ and the perforation rate was $56.3 \%$. Some papers reported that the mortality for complicated $\mathrm{OH}$ was $12-70 \%$ [11]. Thus, in principle, essentially all patients must undergo surgery, whether laparotomy or a laparoscopic operation [6]. Furthermore, many patients undergo surgery to prevent the recurrence of $\mathrm{OH}$. Because we overlooked her $\mathrm{OH}$, our patient happened to be treated conservatively without surgery. Fortunately, her ileus resolved by decompression with an ileus tube without any complications. Even with surgery, postoperative complications occur in some cases; most complications are cardiac or pulmonary in origin [3]. Morbidity and mortality of $\mathrm{OH}$ tend to be high because

\section{References:}

1. Nasir BS, Zendejas B, Ali SM et al: Obturator hernia: the Mayo Clinic experience. Hernia, 2012; 16: 315-19

2. Igari $\mathrm{K}$, Ochiai T, Aihara $\mathrm{A}$ et al: Clinical presentation of obturator hernia and review of the literature: Hernia, 2010; 14: 409-13 most patients are aged and have comorbidities such as chronic obstructive pulmonary disease, chronic renal failure, or ischemic heart disease. This is why early diagnosis by abdominal CT cannot necessarily improve the morbidity and mortality of $\mathrm{OH}[3,7]$. In addition, cooperation during medical procedures is often difficult to obtain because of dementia, as in our patient. Fortunately, our patient improved without any residual problems or recurrences despite our oversight on abdominal CT.

\section{Conclusions}

In conclusion, when a thin, elderly, female patient has ileus, regardless of the absence of history of abdominal surgery, it is imperative to consider the possibility of $\mathrm{OH}$ because early diagnosis is the key to the survival of the patient. Although abdominal $\mathrm{CT}$ is an excellent modality, the diagnosis of $\mathrm{OH}$ can be elusive when its findings are small and subtle, so careful reading is mandatory.

\section{Conflict of interest statement}

The authors state that they have no conflicts of interest to declare. 
5. Cai X, Song X, Cai X: Strangulated Intestinal Obstruction Secondary to a Typical Obturator Hernia: A case Report with Literature Review. Int J Med Sci, 2012; 9: 213-15

6. Katoh T, Kawamoto R, Kusunoki T: A case of obturator hernia with overlooked Howship-Romberg sign and treated as sciatica. Nihon Ronen Igakkai Zasshi, 2011; 48: 176-79

7. Yokoyama Y, Yamaguchi A, Isogai M et al: Thirty-six Cases of Obturato Hernia: Does Computed Tomography Contribute to Postoperative Outcome? World J Surg, 1999; 23: 214-17

8. Nakayama T, Kobayashi S, Shiraishi K et al: Diagnosis and treatment of obturator hernia. Keio J Med, 2002; 51: 129-32
9. Saibeni S, Rondonotti E, lozzelli A et al: Imaging of the small bowel in Crohn's disease: A review of old and new techniques. World J Gastroenterol, 2007, 13: $3279-87$

10. Terada R, Ito S, Kidogawa $\mathrm{H}$ et al: Obturator hernia: The usefulness of emergent computed tomography for early diagnosis. J Emerg Med, 1999; 17(5): 883-86

11. Rodriguez-Hermosa Jl, Codina-Cazador A, Maroto-Genover A et al: Obturator hernia: clinical analysis of 16 cases and algorithm for its diagnosis and treatment. Hernia, 2008; 12: 289-97

12. Leow JJ, How KY, Goh MH et al: Non-operative management of obturator hernia in an elderly female: Hernia 2013; [Epub ahead of print] 